\title{
Influence of Ramp Position on Joint Biomechanics During Elliptical Trainer Exercise
}

\author{
Kathleen M. Knutzen ${ }^{1,2, *}$ Wren L. McLaughlin ${ }^{1}$, Andrew J. Lawson ${ }^{1}$, Brandi S. Row ${ }^{1}$ and \\ LeaAnn Tyson Martin ${ }^{1}$ \\ ${ }^{I}$ Western Washington University, Department of Physical Education, Health and Recreation, 516 High Street, \\ Bellingham, WA 98225-9067, USA \\ ${ }^{2}$ California State University, Bakersfield, School of Social Sciences and Education, 9001 Stockdale Highway, \\ Bakersfield, CA 93311, USA
}

\begin{abstract}
Introduction: The elliptical trainer, developed to simulate running while minimizing joint loads, elicits a unique lower extremity biomechanical response. The purpose of this study was to examine the angular kinematics, peak net joint moments, and peak joint powers at the hip, knee and ankle joints while exercising at three different ramp settings on the elliptical trainer exercise machine (Precor EFX).

Methods: Twenty-six healthy individuals with no history of lower extremity injury and with previous experience exercising on an elliptical trainer volunteered for this study. Motion was captured with two cameras as subjects performed exercise at three ramp conditions. The pedal resistance was kept constant at the lowest setting. The pedals of the elliptical were fitted with three orthogonal load cells. Video and force data were synchronized and used to perform a 2D inverse dynamics analysis.

Results: As the ramp inclination increased, subjects demonstrated greater amounts of ankle dorsiflexion, knee flexion, hip flexion and lesser degrees of plantar flexion and hip extension ( $\mathrm{p} .000)$. Mean peak moments at the ankle joint were not significantly different across the ramp settings, but peak knee extensor and hip flexor moments increased while knee flexor and hip extensor at the hip decreased $(\mathrm{p}<.000)$. As the ramp inclination increased, peak positive power at both the knee and hip significantly decreased and negative power decreased at the knee joint $(p<.000)$, though the effect size for these changes was small.

Conclusions: The hip and knee joints were observed as the primary sources of power during exercise on the elliptical trainer at the lowest resistance setting.
\end{abstract}

Keywords: Exercise Equipment, Lower Extremity, Joint Kinetics, Joint Kinematics.

\section{INTRODUCTION}

It has been estimated that between $65-70 \%$ of runners will sustain an overuse injury in their lower extremities [1]. Gait simulators, such as the elliptical trainer, were developed to replicate walking or running while keeping the user suspended on moving foot pedals. As the foot remains in contact with the pedal throughout the entire motion, impact forces are minimized. Previous research has focused on the physiological response to exercise on the elliptical trainer, showing that it produces similar cardiorespiratory responses, such as maximal oxygen consumption, to treadmill running [2-3]. However, little is known about the biomechanics of the elliptical stride, with very few studies evaluating how closely the elliptical trainer actually mimics the kinematics and kinetics of running or walking [4].

With an elliptical trainer, the ankle joint follows an elliptical path and hence, the lower extremity movement pattern is primarily determined by the machine. The elliptical path

*Address correspondence to this author at the California State University, Bakersfield, School of Social Sciences and Education, 9001 Stockdale Highway, Bakersfield, CA 93311, USA; Tel: 661-654-2201;

E-mail: kknutzen@csub.edu can be modified by adjusting the angle of incline and the workload can be modified by adjusting the resistance to motion on some models of the elliptical trainer. While impact forces are minimized as the feet remain in contact with the pedal through the entire stride, not all the stresses on the joints originate from impact forces. Forces generated by muscles to move the body through the path enforced by the elliptical trainer and external moments generated by the elliptical trainer cause internal loads on the joints of the lower extremities.

Furthermore, some elliptical trainers enlist arm levers which may significantly change the mechanics and force application throughout the elliptical stride.

Investigations on the lower extremity movements and forces produced during exercise on the elliptical trainer have shown the closed-chain elliptical motion does produce lower impact forces than treadmill running, and peak vertical reaction forces appear to be similar during elliptical exercise and walking [4]. Although the specific elliptical trainer model and exercise bout machine settings were not reported, Porcari, Zedaker, Naser, and Miller [4-6] found that pedal reaction forces (PRF) during elliptical exercise were similar to treadmill walking but not running with sixteen adult subjects at a 
self-selected pace. Lu, Chien and Chen and Lu and Chien [4, 5] examined overground walking and elliptical striding of male adults. Mean peak vertical PRF was less for the elliptical than mean vertical ground reaction force (GRF) walking. Additionally, Lu and Chien [5] found that PRF in the posterior portion of the elliptical stride (pedal behind the midline of the body) were significantly larger for elliptical striding as opposed to overground walking, though the cadence was slow (50 strides per minute), and the presence or absence of arm levers was not indicated.

While the elliptical may have been designed to simulate normal gait kinematics, preliminary evidence on lower extremity joint angles and joint moments modeled using inverse dynamics does not support these claims. Lu and Chien [5] found peak flexion angles at the hip, knee, and ankle and peak hip flexor and knee extensor moments on the elliptical were greater than those during walking. Additionally, peak hip extensor, knee flexor, and ankle plantar flexor moments on the elliptical were smaller than during walking. These differences were most likely due to the fixed pedal position [5]. Chien, Tsai, and Lu [7] also investigated the effect of stride length and power outputs on lower extremity biomechanics during exercise on the elliptical trainer. Peak ankle plantar flexion angle increased for all workloads at the longer stride length. Peak hip flexor, knee extensor, and ankle dorsiflexor moments increased while peak ankle plantar flexor moment decreased. When comparing both stride lengths to different workloads, the peak ankle dorsiflexor, knee flexor, knee extensor, and hip flexor moments increased significantly with increasing workload.

Unsubstantiated claims regarding benefits of the elliptical machine include the similarity of the elliptical and walking/running stride, and the lack of risk for injury to the back, ankles, knees or hips. Critics of the elliptical trainer have expressed concerns that the stride feels unnatural and could expose the knee to harmful loads, resulting in injury or reinjury if the elliptical trainer was prescribed for rehabilitation. The elliptical stride can be modified by adjusting the ramp setting, and it is feasible that modifying the degree of ramp incline influences the loading of the lower extremity joints. The purpose of this study was to perform a biomechanical analysis of the lower extremity joints while striding on an elliptical trainer at three ramp settings. Specifically, the relative joint (ankle, knee, hip) angles, peak vertical and anteroposterior joint forces, net joint moments, and joint powers were quantified.

\section{METHODS}

\section{Subjects}

Twenty-six (20 female, 6 male) college-aged subjects (Mean (SD) age $22.2(0.85)$ yr; height $169.8(9.75) \mathrm{cm}$; mass $74.0(19.3) \mathrm{kg}$ ) participated in this study after being informed of all the procedures and risks associated with the study. The Human Subjects Review Committee at Western Washington University approved all methods and procedures, and written informed consent was obtained from each subject prior to testing. The activity level for the subjects ranged from active to very active and all subjects had prior experience using an elliptical trainer and did not require a familiarization trial.

\section{Instrumentation}

Subject trials were performed on a commercially available Precor EFX 546 elliptical fitness crosstrainer (Precor, Woodinville, WA). The elliptical has an inclination range of 13-40 degrees (CrossRamp ${ }^{\circledR}$ setting 3-20) and a resistance setting range from levels 1 to 20 (lowest setting used). The model did not have arm levers; thus the movement of the pedals was a result of the lower extremities only. Kinematic data were collected using a two camera motion analysis system (Qualisys, Inc., Glastonbury, CT) which recorded at a frequency of $60 \mathrm{~Hz}$. Pedal reaction forces of the right pedal were measured using three orthogonal strain gauges mounted in the pedal plate. Forces were measured in the X, Y, and Z axis at a frequency of $600 \mathrm{~Hz}$. The cameras and the pedal forces were all interfaced and collected with the same microcomputer.

\section{Experimental Study Design and Procedures}

A within-subjects design was utilized in this study as each participant completed an exercise bout on the elliptical trainer at three different ramp settings. The subjects were instrumented with retroreflective markers placed on the following bony landmarks on the right side of the body: the foot (head of $1^{\text {st }}$ metatarsal, lateral aspect of $5^{\text {th }}$ metatarsal head, and calcaneus); the right lateral malleolus at the ankle; the lateral condyle of the femur at the knee; the greater trochanter of the femur; and the anterior-superior aspect of the iliac spine. Since the subjects wore athletic shoes, the lateral aspect of the $5^{\text {th }}$ metatarsal head was palpated through the shoe and the marker was placed on the shoe. The calcaneus marker was placed on the lateral heel of the shoe. These points allowed for the tracking of the motion of the lower limb segments during the elliptical stride. Three additional markers were placed on the anterior and posterior ends of the right footplate and directly over the origin of the three pedal transducers. Body segment length parameters were also determined for the thigh, shank, and foot. Using previously published standards, thigh, shank, and foot mass and moment of inertia could then be determined [8].

Testing was preceded by a 5 -minute warm-up period on the elliptical trainer. After the warm-up period, subjects were instructed to begin striding on the elliptical at a stride rate of 120 strides per minute, as measured by a metronome. Subjects were instructed to maintain this pace throughout their respective testing period. Each data collection session consisted of approximately 10 minutes total on the elliptical trainer, 2 minutes each at three progressive ramp settings: 13, 25, and 40 degree incline (CrossRamp ${ }^{\circledR}$ levels 03, 12, and 20 , respectively), as measured by the machine. These inclines represented the manufacturer's baseline ramp setting, the maximum ramp setting, and a midway ramp setting. The resistance was set to the lowest setting and maintained at that setting for the duration of the data collection period. A standardized resistance setting was utilized to remove the confounding influence of resistance from the analysis and to provide a resistance that would allow all subjects to complete all ramp trials. Data collection with both kinematic and kinetic equipment began after 30 consecutive seconds of striding at the determined pace and continued for 10 seconds. At two minutes, the ramp was increased to the next setting ( 25 or 40 degrees) and each subject was given 30 seconds to 
ensure pace maintenance before the 10 second data collection commenced.

\section{Measurements}

Segment angles, joint angles, as well as linear and angular segmental velocities and accelerations were calculated from filtered joint marker position data. The identification of the segment and joint angles are shown in Fig. (1). Since the kinematic and kinetic data were synchronized yet collected at different frequencies, the kinetic data was scaled to match the time domain of $60 \mathrm{~Hz}$. Prior to inverse dynamics calculations, all raw data were filtered at $8 \mathrm{~Hz}$ using a criticallydamped, $4^{\text {th }}$ order, recursive, low pass Butterworth filter. Calculations were performed using a customized Microsoft Excel spreadsheet.

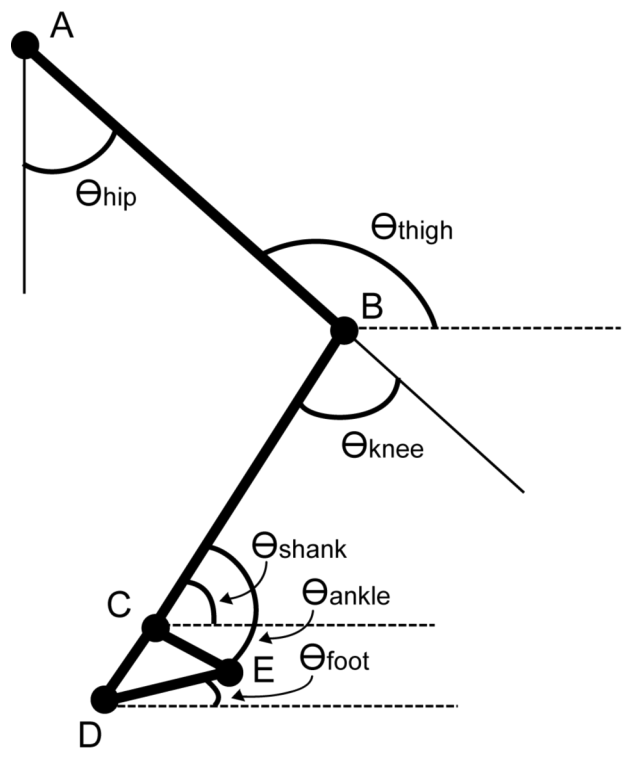

Fig. (1). Segment angles and relative joint angles of the lower extremity. Reflective markers are identified as follows; greater trochanter of femur (A), lateral condyle of femur (B), lateral malleolus of fibula (C), calcaneus (D), and head of $5^{\text {th }}$ metatarsal (E). The dashed line indicates right horizontal.

Foot, shank, and thigh segment masses were calculated as a proportion of total body mass using Dempster's parameters. The moment of inertia around the center of mass was computed using standard equations [9]. Joint reaction forces and moments were calculated at the ankle, knee and hip using the Newton-Euler inverse dynamics approach. Segmental masses and moments of inertia, segment position data, segment linear and acceleration data, as well as vertical and horizontal pedal reaction force data were used in the calculations. The lower extremity was modeled as a three segment planar system with external reaction forces located at the distal end of the foot segment on the pedal (Fig. 2). An assumption that the center of pressure for the resultant pedal reaction force was directly over the transducer marker was made. To standardize foot placement, all subjects placed their feet on the pedals with the front edge of their shoes up against the front edge of the pedal. Maximal joint reaction forces (vertical/anterior/posterior), joint moments (flexor/ extensor) and power (positive/negative) values were determined for the sampling interval of three stride cycles. Power was calculated by taking the cross-product of the angular velocity and joint moment at each time interval. Total power was calculated by summing the three joint powers. This process was repeated at the hip, knee, and ankle for each of the three ramp conditions. Forces were normalized to body weight (BW) in Newton and moments and powers were normalized to body mass in kilograms.



Fig. (2). Lower extremity linked-segment model. Pedal reaction forces (PRF) shown occur during the stance phase of elliptical stride.

Events in the elliptical stride were determined using potentiometer data in the instrumented pedal. The movement cycle was divided into two phases (Fig. 3). The stance phase was defined as the phase of the elliptical that begins with the most anterior pedal position and ends at the most posterior pedal position. The closed chain swing phase was identified as beginning at the most posterior pedal position and ending at the most anterior pedal position.

The relative joint angles at which the peak kinetic values occurred were calculated at each joint. A determination of whether the peak values occurred during the closed-chain swing phase or support phase of the elliptical stride was also made using potentiometer data from the instrumented pedal. For the three ramp conditions, values over time for a representative subject were normalized to $100 \%$ for the stance phase and closed-chain swing phase, separately. The three ramp conditions were then displayed graphically to illustrate the study findings and provide information regarding when peaks occur with respect to the motion phases. 


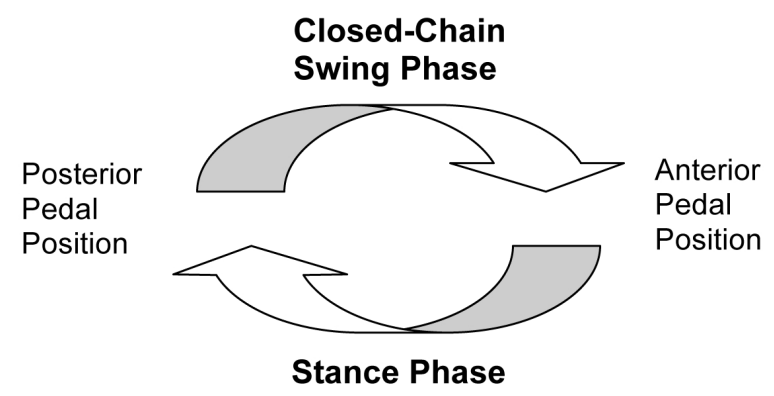

Fig. (3). Phases of the elliptical stride. Arrows indicate direction of motion.

\section{Statistical Analysis}

Repeated-measures, one-way analysis of variance (ANOVA) was used to determine if significant differences existed between different ramp conditions. In addition to the relative joint angles, normalized peak vertical forces, anterior and posterior forces, flexor and extensor moments and positive and negative joint powers at the ankle, knee, and hip were used in the analysis. Total power was also tested for statistical significance across the three ramp conditions. A Bonferroni correction was applied to account for the multiple ANOVA tests run. Significance was defined as a $p$-value less than .002. When significant main effects were found, planned contrasts between the three ramp conditions were performed. A Bonferroni correction to account for the multiple contrasts performed was applied and $p$-values of less than .017 were considered significant. The meaningfulness of the data were determined by calculating omega squared $\left(\omega^{2}\right)$ for the main ANOVA and Pearson's correlation coefficients $(r)$ for the individual contrasts. Calculations were performed using Microsoft EXCEL and SPSS Statistical Software Package Version 13.0 (SPSS Inc., Chicago, IL.).

\section{RESULTS}

\section{Kinematic Parameters}

Overall, the relative joint angles of the lower extremity changed significantly as the ramp setting increased. Table $\mathbf{1}$ shows the mean peak relative angles for the ankle, knee, and hip joints. As the ramp inclination increased, the ankle joint moved through a greater degree of dorsiflexion while the degree of plantarflexion was reduced. Approximately $30 \%$ of the differences in peak ankle motion are attributable to the ramp setting. Contrasts revealed large effects for both dorsiflexion and plantarflexion across the three ramp settings (contrast, $r=$ dorsiflexion \& plantarflexion; low vs. middle, $r$ $=.86 \& .91$, middle vs. high, $.86 \& .68$, low vs. high, $.90 \&$ $.92)$. Knee flexion increased significantly from low to high ramp settings. An $18 \%$ increase (12.4 deg) in knee flexion was observed between the low and high settings. The knee did not reach a fully extended position during the elliptical stride nor were there significant differences in knee extension across the ramp conditions. The magnitude of peak hip flexion showed the greatest change across the ramp settings. Hip flexion increased 37\% (16 deg) between the low and high settings, with a $20 \%$ increase ( 9 deg) observed between the low and middle ramp settings and a 13\% increase (7.6 deg) observed between the middle and high settings. The ramp setting explains $81 \%$ of the observed variance in hip flexion. The hip joint moved through a greater extension range during the low ramp setting and while contrasts revealed large effects with comparing the low to the middle ( $r$ $=.94)$ and the low to the high $(r=.91)$ setting, only moderate effects were observed between the middle and high $(r=$ .50) ramp setting. A representative sample of relative joint angles across the stride cycle is shown in Fig. (4). Peak ankle dorsiflexion occurred during the stance phase and peak plantarflexion occurred during the closed-chain swing phase. Qualitative analysis of temporal patterns of the ankle joint motion revealed that there was more variability at the ankle joint than at the knee and hip joints.

The pattern and timing of the knee and hip joint motion are very similar. Peak knee and hip extension occurred during the last quarter of the stance phase, while peak knee and hip flexion occurred during the final quarter of the closedchain swing phase. The range of peak knee flexion was 61 to 90 degrees and the range for peak extension was 0 to 30 degrees of flexion across all ramp settings and subjects. The

Table 1. Means (SD) for Peak Relative Joint Angles (Degrees) Across Three Ramp Settings

\begin{tabular}{|c|c|c|c|c|c|}
\hline Ramp & Low & Middle & High & $p$-value & $\omega^{2}$ \\
\hline \multicolumn{6}{|l|}{ Ankle } \\
\hline Dorsiflexion $^{\mathrm{b}, \mathrm{c}, \mathrm{d}}$ & $-1.30(5.11)$ & $2.23(4.59)$ & $7.21(5.48)$ & $.000^{\mathrm{a}}$ & .32 \\
\hline Plantarflexion ${ }^{\mathrm{b}, \mathrm{c}, \mathrm{d}}$ & $19.40(6.44)$ & $12.99(5.97)$ & $9.52(5.92)$ & $.000^{\mathrm{a}}$ & .31 \\
\hline \multicolumn{6}{|l|}{ Knee } \\
\hline Flexion ${ }^{\mathrm{b}, \mathrm{c}, \mathrm{d}}$ & $69.14(4.61)$ & $73.93(5.41)$ & $81.53(4.59)$ & $.000^{\mathrm{a}}$ & .52 \\
\hline Extension $^{*}$ & $14.91(7.40)$ & $17.44(6.25)$ & $17.47(6.76)$ & .002 & .03 \\
\hline \multicolumn{6}{|l|}{ Hip } \\
\hline Flexion $^{\mathrm{b}, \mathrm{c}, \mathrm{d}}$ & $45.63(2.89)$ & $54.76(3.23)$ & $62.36(3.73)$ & $.000^{\mathrm{a}}$ & .81 \\
\hline Extension $^{\mathrm{b}, \mathrm{c}, \mathrm{d}}$ & $6.78(3.73)$ & $3.06(3.65)$ & $1.90(3.66)$ & $.000^{\mathrm{a}}$ & .24 \\
\hline
\end{tabular}

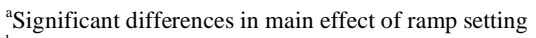

${ }^{b}$ Low ramp setting significantly different from middle ramp setting at $p \leq 0.017$

${ }^{\mathrm{c}}$ Middle ramp setting significantly different from high ramp setting at $p \leq 0.017$

${ }^{\mathrm{d}}$ Low ramp setting significantly different from high ramp setting at $p \leq 0.017$

"Positive values indicate minimum flexion angle. 


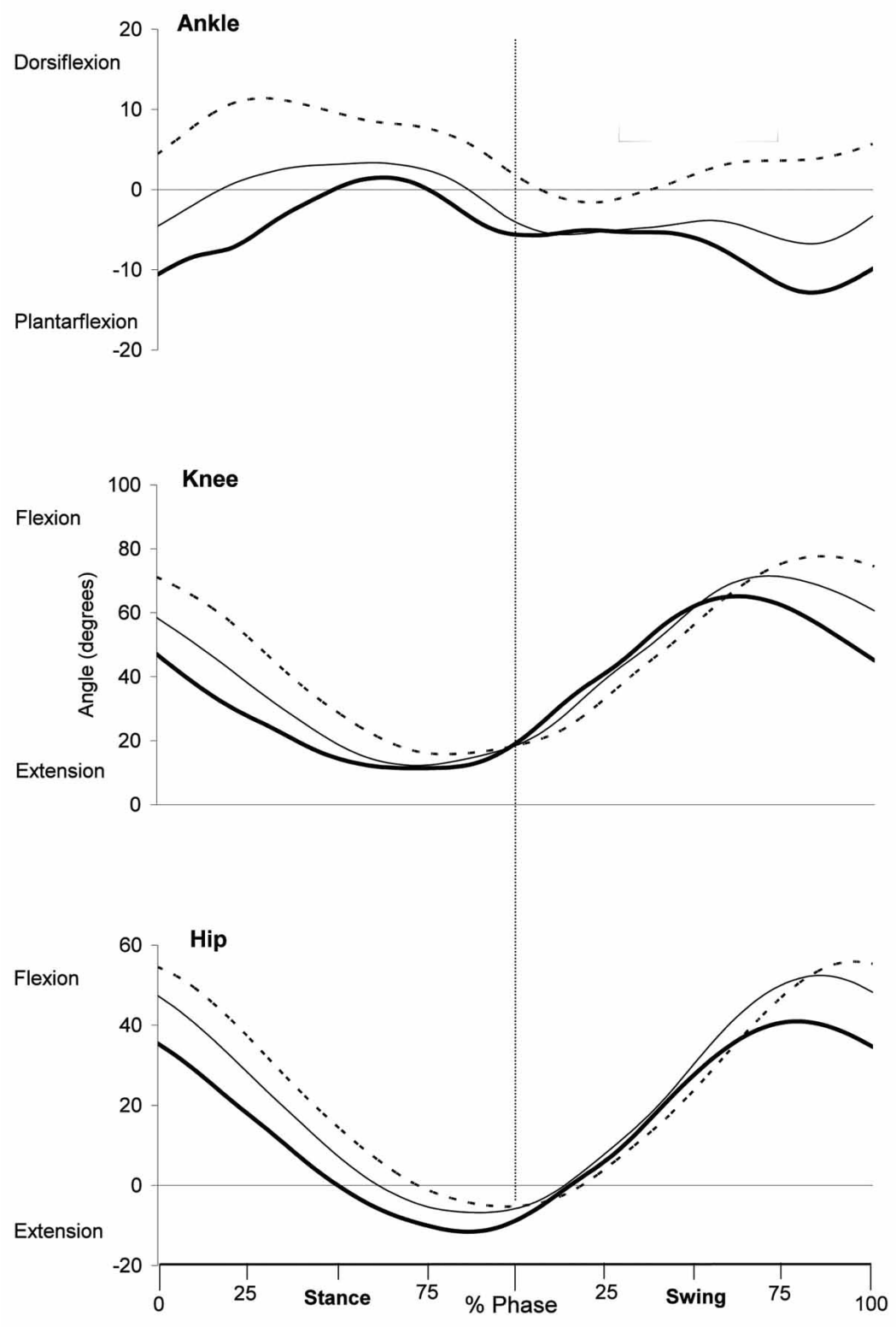

Fig. (4). Representative sample of relative joint angles for one stride cycle. Low (bold black), middle (black), and high (dashed) ramp conditions are shown. Vertical dashed line indicates change from stance to closed swing phase.

range for peak hip flexion was 41 to 76 degrees and the range for peak hip extension was 14 degrees of hyperextension to 4 degrees of flexion. As the ankle is dorsiflexing during the stance phase, the knee and the hip are extending. During the closed-chain swing phase, when the ankle is plantarflexing, the hip and knee are flexing. These observations indicate that the motion of the elliptical stride is distinct from gait.

\section{Joint Forces}

Joint reaction forces oriented in the sagittal plane were determined for the ankle, knee, and hip across the three ramp conditions. Anteroposterior joint reaction forces were represented as the resultant force in the $\mathrm{X}$ direction, with positive values indicating anteriorly directed and negative values indicating posteriorly directed forces; at the knee, an anterior joint reaction force would result in the anterior translation of the tibia with respect to the femur (as seen in Fig. (2), where $\mathrm{Kx}$ represents the anterior-posterior knee joint reaction force). As ramp setting increased, peak anterior joint reaction forces increased significantly while posterior joint reaction forces decreased significantly at all three joints (Table 2). Anterior reaction forces increased by $40 \%$, while posterior reaction forces decreased by 71 to $100 \%$ between the low and high settings. Contrasts revealed meaningful differences between all ramp conditions (contrast, $r=$ anterior $\&$ posterior; low vs. middle, $r=.78 \& .74$, middle vs. high, $r=.76 \&$ .54 , low vs. high, $r=.92 \& .82$ ). Fig. (5) shows the anteroposterior joint reaction forces at the knee for a representative subject. The ankle and hip display the same pattern over the stride cycle (data not shown). Peak anterior joint reaction forces occur during the second half of the stance phase. The knee and hip were at or near end extension range and the ankle was dorsiflexed when the peak anterior joint reaction forces occurred. During the early to mid closed-chain swing phase, the anteroposterior forces are minimal and then the peak posterior force occurs near the anterior pedal position, at the end of the closed-chain swing phase. The knee and hip 
Table 2. Means (SD) for Peak Forces (BW) for Three Ramp Conditions

\begin{tabular}{|c|c|c|c|c|c|}
\hline Ramp & Low & Middle & High & $p$-value & $\omega^{2}$ \\
\hline \multicolumn{6}{|l|}{ Ankle } \\
\hline Anterior ${ }^{\mathrm{b}, \mathrm{c}, \mathrm{d}}$ & $0.26(0.03)$ & $0.30(0.04)$ & $0.36(0.05)$ & $.000^{\mathrm{a}}$ & .54 \\
\hline Posterior $^{\mathrm{b}, \mathrm{c}, \mathrm{d}}$ & $0.10(0.03)$ & $0.07(0.03)$ & $0.05(0.03)$ & $.000^{\mathrm{a}}$ & .41 \\
\hline Vertical & $1.10(0.15)$ & $1.14(0.17)$ & $1.19(0.23)$ & .010 & .03 \\
\hline \multicolumn{6}{|l|}{ Knee } \\
\hline Anterior $^{\mathrm{b}, \mathrm{c}, \mathrm{d}}$ & $0.26(0.03)$ & $0.31(0.04)$ & $0.37(0.05)$ & $.000^{\mathrm{a}}$ & .54 \\
\hline Posterior $^{b, c, d}$ & $0.11(0.03)$ & $0.08(0.03)$ & $0.06(0.03)$ & $.000^{\mathrm{a}}$ & .40 \\
\hline Vertical $^{\mathrm{b}, \mathrm{d}}$ & $0.93(0.15)$ & $0.82(0.16)$ & $0.77(0.19)$ & $.000^{\mathrm{a}}$ & .12 \\
\hline \multicolumn{6}{|l|}{ Hip } \\
\hline Anterior $^{\mathrm{b}, \mathrm{cd}}$ & $0.27(0.03)$ & $0.32(0.04)$ & $0.38(0.05)$ & $.000^{\mathrm{a}}$ & .54 \\
\hline Posterior $^{b, c, d}$ & $0.12(0.03)$ & $0.09(0.03)$ & $0.07(0.03)$ & $.000^{\mathrm{a}}$ & .39 \\
\hline Vertical $^{\mathrm{b}, \mathrm{d}}$ & $0.82(0.14)$ & $0.71(0.16)$ & $0.66(0.19)$ & $.000^{\mathrm{a}}$ & .13 \\
\hline
\end{tabular}

${ }^{\text {a }}$ Significant differences in main effect of ramp setting

${ }^{\mathrm{b}}$ Low ramp setting significantly different from middle ramp setting at $p \leq 0.017$

${ }^{\mathrm{c}}$ Middle ramp setting significantly different from high ramp setting at $p \leq 0.017$

${ }^{\mathrm{d}}$ Low ramp setting significantly different from high ramp setting at $p \leq 0.017$

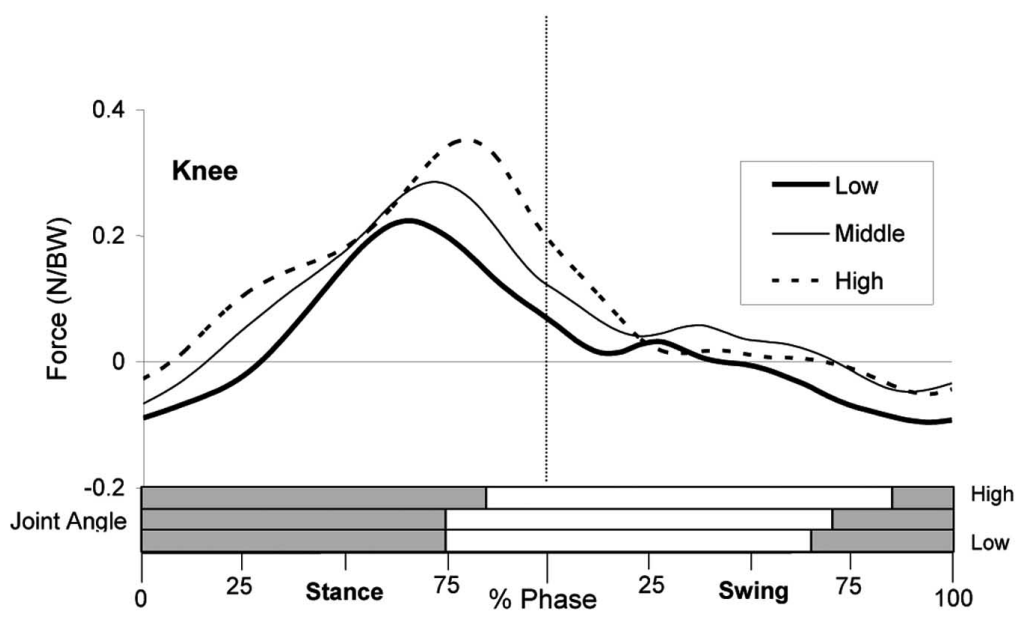

Fig. (5). Representative sample of knee anteroposterior joint reaction forces for one stride cycle. Low (bold black), middle (black), and high (dashed) ramp conditions are shown. Vertical dashed line indicates change from stance to closed swing phase. Shaded bars indicate when leg is extending at the knee joint, and white bars indicate when leg is flexing at the knee joint. Shade transitions indicate position of minimum and maximum joint angles.

were flexed and the ankle was plantarflexed when the peak posterior joint reaction forces occurred.

At the ankle joint, vertical joint reaction forces did not differ significantly across the three ramp conditions (Table 2). At the hip and the knee, vertical joint reaction forces decreased in magnitude as the ramp inclination increased. While contrasts revealed meaningful differences between the low and middle and low and high settings, only 12 and $13 \%$ of the observed variance was attributable to the ramp at the knee and hip, respectively. Fig. (6) shows the vertical reaction forces for a representative subject over one stride cycle. Peak vertical forces occurred during the second half of the stance phase when the ankle was plantarflexing and knee and hip were extending. In most subjects, the peak forces occurred later in the stance phase as the ramp setting moved from low to high. The joint reaction forces were minimal throughout the closed-chain swing phase and began to increase again as the pedal approached the anterior position.

\section{Net Joint Moments}

Table 3 shows the mean peak net joint moments at the ankle, knee, and hip joints. Mean peak net joint moments at the ankle joint were not significantly different across the three ramp settings. At the knee joint, the peak extensor moment increased significantly as the ramp setting increased. Contrasts reveal meaningful differences between the middle and high $(r=.62)$ and the low and high $(r=.68)$ but not between the low and middle conditions $(r=.18)$. Flexor moments at the knee decreased as ramp incline increased. The ramp setting accounted for $85 \%$ of the observed differ- 


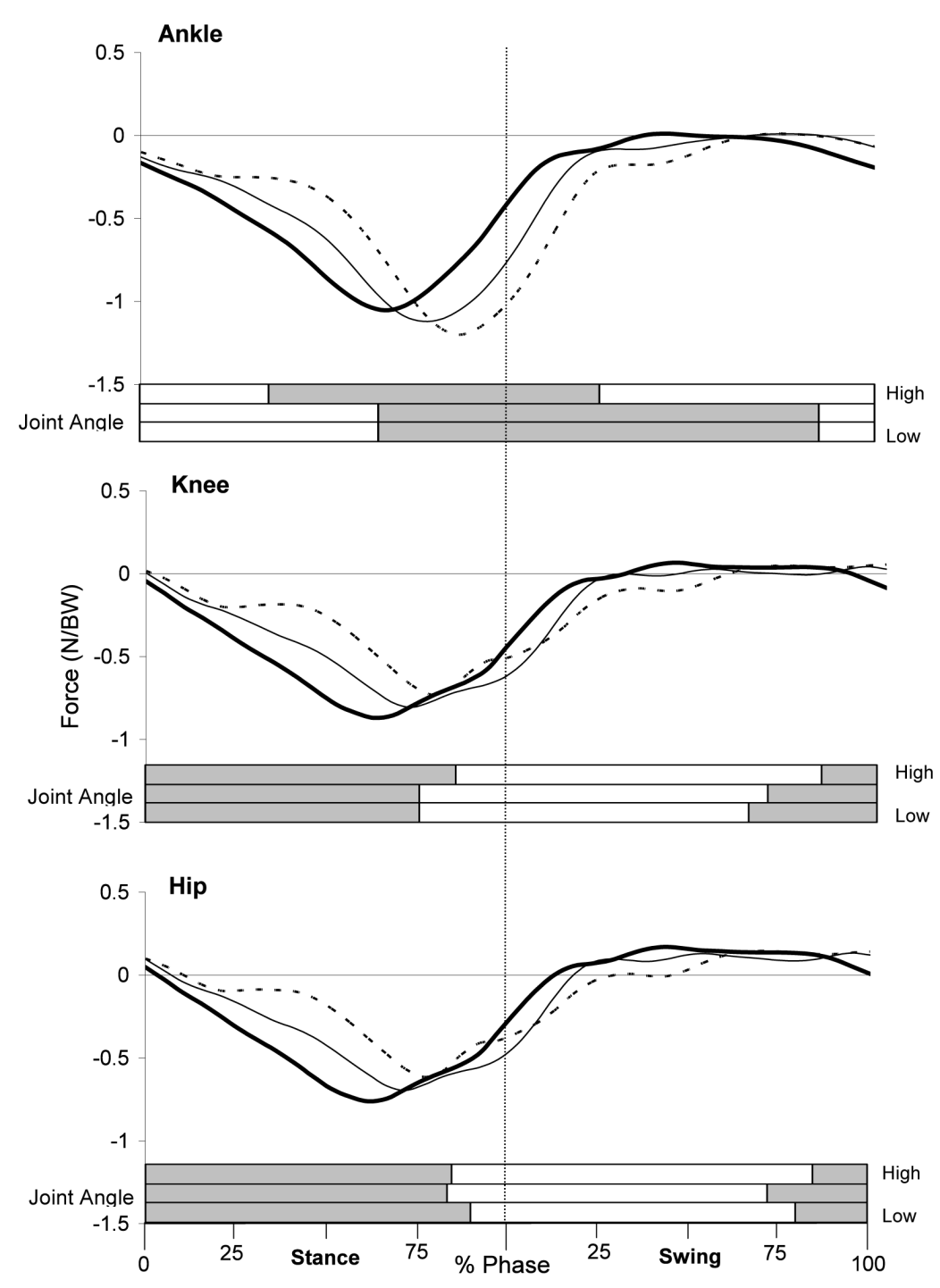

Fig. (6). Representative sample of the vertical joint reaction forces for one stride cycle. Low (bold black), middle (black), and high (dashed) ramp conditions are shown. Vertical dashed line indicates change from stance to closed swing phase. Shaded bars indicate when segment is extending (or plantarflexing) at the joint, and white bars indicate when segment is flexing (or dorsiflexing) at the joint. Shade transitions indicate position of minimum and maximum joint angles.

ences in flexor moments, and meaningful differences were found between all three conditions (low vs. middle, $r=.98$, middle vs. high, .96, low vs. high, .98). The knee flexor moment decreased $96 \%$ from the low to the middle setting and $363 \%$ from the low to the high setting. The pattern of peak hip extensor moments was similar to the knee flexor moments across the ramp settings. As the ramp setting increased, hip extensor moments decreased significantly. Contrasts revealed meaningful differences between all three conditions (low vs. middle, $r=.98$, middle vs. high, .96, low vs. high, .98) and $87 \%$ of the observed variance was explained by the change in the ramp setting. The hip flexor moment, like the knee extensor moment, increased significantly with the ramp incline. While differences were found between all conditions (low vs. middle, $r=.69$, middle vs. high, .81 , low vs. high, .87) the ramp setting could only account for $49 \%$ of the overall variance. Fig. (7) shows the net joint moments from a representative elliptical stride cycle across the three ramp conditions. At the ankle joint, the peak plantarflexor moment occurred during the second half of the stance phase while the ankle was plantarflexing. A large flexor moment at the knee and extensor moment at the hip were observed during stance phase while both the knee and the hip were extending. As the ramp setting increased, the peak knee flexor moment was reduced and a small knee extensor moment was generated. At the hip, the peak extensor moment was reduced and a larger flexor moment was generated as ramp inclination increased. These knee extensor/hip flexor moments occurred at the beginning of the closed-chain swing phase. At this time, both the hip and knee are flexing; the motion is determined by the pedals moving in the elliptical pattern. As the ramp incline increases, the orientation of the ellipse that the leg segment must be propelled through the first half of the closed-chain swing phase is steeper and therefore, a larger knee extensor moment is observed while bringing the leg forward during early closed-chain swing. The hip flexor moment assists the lower limb with the upward motion during the closed-chain swing phase. 
Table 3. Means (SD) for Peak Net Joint Moments (Nm/kg) for Three Ramp Conditions

\begin{tabular}{|c|c|c|c|c|c|}
\hline Ramp & Low & Middle & High & $p$-value & $\omega^{2}$ \\
\hline \multicolumn{6}{|l|}{ Ankle } \\
\hline Plantarflexor & $0.37(0.20)$ & $0.34(0.22)$ & $0.41(0.30)$ & .022 & .01 \\
\hline Dorsiflexor & $0.12(0.09)$ & $0.08(0.06)$ & $-0.07(0.06)$ & .002 & .07 \\
\hline \multicolumn{6}{|l|}{ Knee } \\
\hline Extensor $^{\mathrm{c}, \mathrm{d}}$ & $0.05(0.12)$ & $0.09(0.15)$ & $0.28(0.23)$ & $.000^{\mathrm{a}}$ & .24 \\
\hline Flexor $^{\mathrm{b}, \mathrm{c}, \mathrm{d}}$ & $2.27(0.43)$ & $1.16(0.26)$ & $0.49(0.18)$ & $.000^{\mathrm{a}}$ & .85 \\
\hline \multicolumn{6}{|l|}{ Hip } \\
\hline Extensor $^{\mathrm{b}, \mathrm{c}, \mathrm{d}}$ & $2.08(0.39)$ & $0.94(0.23)$ & $0.27(0.18)$ & $.000^{\mathrm{a}}$ & .87 \\
\hline Flexor $^{\mathrm{b}, \mathrm{c}, \mathrm{d}}$ & $0.20(0.12)$ & $0.29(0.08)$ & $0.56(.22)$ & $.000^{\mathrm{a}}$ & .49 \\
\hline
\end{tabular}

${ }^{a}$ Significant differences in main effect of ramp setting

${ }^{b}$ Low ramp setting significantly different from middle ramp setting at $p \leq 0.017$

${ }^{c}$ Middle ramp setting significantly different from high ramp setting at $p \leq 0.017$

${ }^{\mathrm{d}}$ Low ramp setting significantly different from high ramp setting at $p \leq 0.017$

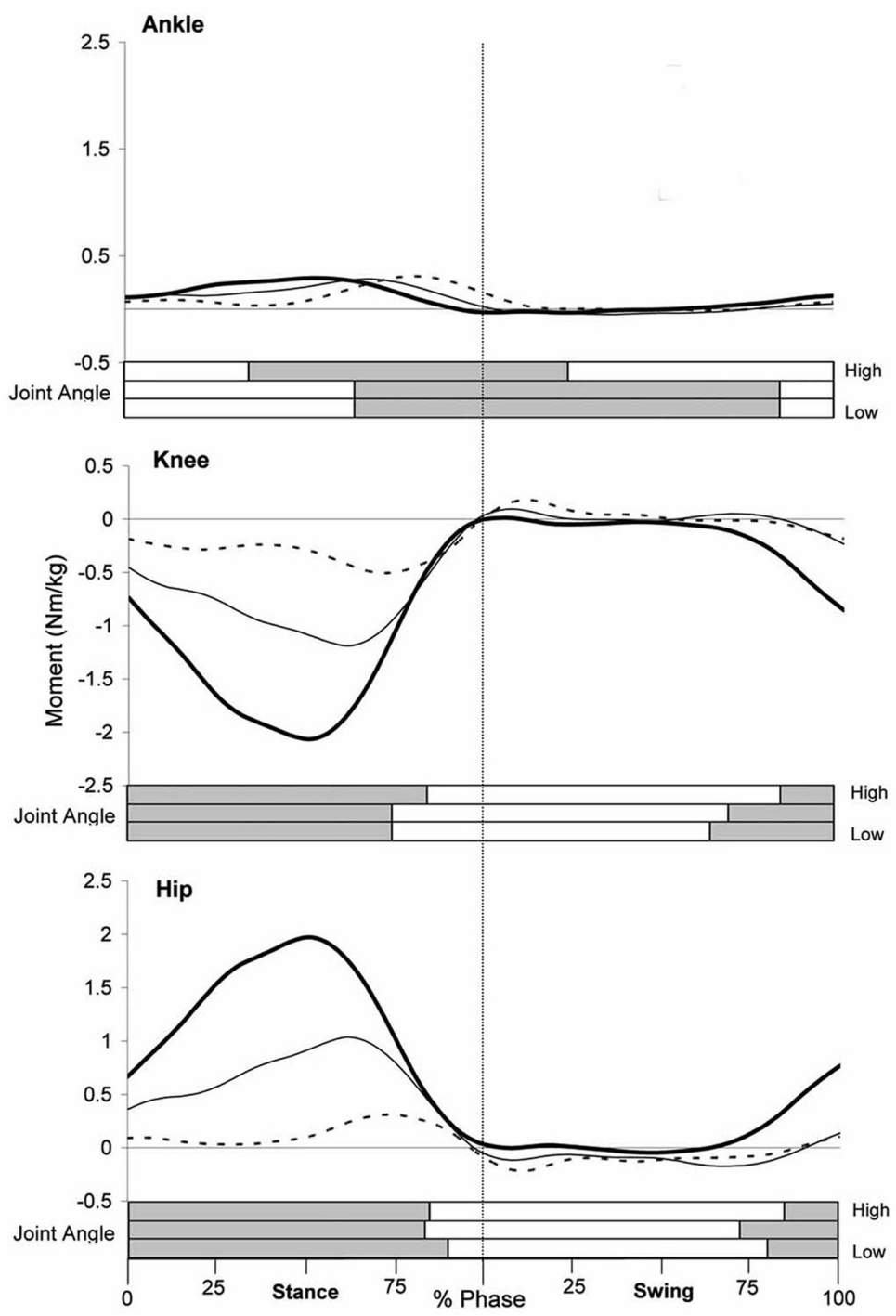

Fig. (7). Representative sample of the net joint moments for one stride cycle. Positive values are extensor (plantarflexor) moments. Low (bold black), middle (black), and high (dashed) ramp conditions are shown. Vertical dashed line indicates change from stance to closed swing phase. Shaded bars indicate when segment is extending (or plantarflexing) at the joint, and white bars indicate when segment is flexing (or dorsiflexing) at the joint. Shade transitions indicate position of minimum and maximum joint angles. 


\section{Joint Power}

Positive powers indicate energy generation and negative powers indicate energy absorption (Table 4). While both peak positive and negative ankle joint powers reached statistical significance across the three ramp settings, the effect size of the ramp on power generation was small. Meaningful decreases were revealed for power absorption between the low to middle $(r=.85)$ and low to high $(r=.82)$ ramp settings but not from middle to high $(r=.09)$. Both knee and hip peak power generation decreased as the ramp setting increased. Peak positive knee and hip power decreased 147 $\%$ and $104 \%$ from the low to middle ramp and $183 \%$ and $112 \%$ from the middle to high ramp, respectively. Contrasts revealed meaningful differences between all three ramp conditions for both the knee and the hip joints (contrast, $\mathrm{r}=\mathrm{knee}$ $\&$ hip; low vs. middle, $r=.91 \& .97$, middle vs. high, $.88 \&$ .85 , low vs. high, $.92 \& .97)$ and changes in ramp setting accounted for 64 and $80 \%$ of the variance in power generation observed at the knee and the hip, respectively. Energy absorbed by the knee joint decreased as the ramp setting increased. Meaningful differences occurred between the low and the middle $(r=.91)$ and the low and the high $(r=.87)$ but not between the middle and high ramp settings $(r=.06)$.

Total power (the sum of the individual joint powers) illustrates the overall lower extremity power and the relative contributions of the ankle, knee, and hip joints. By and large, as the ramp setting increased, total peak joint power, both absorbed and generated, decreased. Eighty percent of the observed variance in power generation is explained by the change in ramp while only 21 percent of the observed variance in power absorption is explained by the change in ramp. Contrasts revealed meaningful differences between the three conditions (low vs. middle, $r=.98$, middle vs. high, .85 , low vs. high, .96) for total power generation and meaningful dif- ferences between the low and the middle $(r=.78)$ and the low and the high $(r=.68)$ but not between the middle and high ramp settings $(r=.13)$. Peak power generation occurred during the stance phase and peak power absorption occurred during the last quarter of the swing phase (Fig. 8). The hip made the largest contribution to the total power generated during the elliptical stride and the knee absorbed the most energy. At the low ramp setting, power is generated during hip and knee extension. The ankle's contribution to the positive work done was minimal. As the ramp increased, the amount of hip flexor power increased during the closedchain swing phase.

\section{DISCUSSION}

This present study documented significant sagittal plane kinematic and kinetic changes while exercising using three different ramp settings on the elliptical trainer. In terms of angular kinematics, the joint angles measured at the low ramp setting in the present study were in the range of the elliptical study by Lu, Chien, and Chen [4] and closely matched joint kinematics presented by Burnfield, Shu, Buster, and Taylor [10]. The ramp inclination changed stride mechanics significantly. Movement adjustments for increased ramp settings primarily occurred through an increase in knee and hip flexion and an accompanying decrease in plantarflexion. This increased flexion is most likely due to equipment design as the pedal elevates the leg higher in the upper ramp settings. The magnitude of the relative joint angles occurring at the hip and knee resembled walking at the low ramp and running at the high ramp $[11,12]$ and the knee reaches a more fully extended position during both walking and running as compared to the elliptical trainer [4]. Similar to cycling, the hip and knee move in a simultaneous pattern, extending during the stance phase and flexing during the closed-chain swing phase [13]. The magnitudes and patterns

Table 4. Means (SD) for Peak Joint Power (W/kg) for Three Ramp Conditions

\begin{tabular}{|c|c|c|c|c|c|}
\hline Ramp & Low & Middle & High & $p$-value & $\omega^{2}$ \\
\hline \multicolumn{6}{|l|}{ Ankle } \\
\hline Negative $^{b, d}$ & $0.38(0.11)$ & $0.23(0.09)$ & $0.22(0.12)$ & $.000^{\mathrm{a}}$ & .32 \\
\hline \multicolumn{6}{|l|}{ Knee } \\
\hline Negative $^{b, d}$ & $1.13(0.47)$ & $0.46(0.23)$ & $0.44(0.42)$ & $.000^{\mathrm{a}}$ & .41 \\
\hline \multicolumn{6}{|l|}{ Hip } \\
\hline Positive $e^{b, c, d}$ & $4.25(0.64)$ & $2.08(0.61)$ & $0.98(0.77)$ & $.000^{\mathrm{a}}$ & .80 \\
\hline Negative $^{b, d}$ & $1.09(0.52)$ & $0.58(0.38)$ & $0.64(0.39)$ & $.000^{\mathrm{a}}$ & .21 \\
\hline
\end{tabular}

${ }^{\mathrm{a}}$ Significant differences in main effect of ramp setting

${ }^{\mathrm{b}}$ Low ramp setting significantly different from middle ramp setting at $p \leq 0.017$

${ }^{\mathrm{c}}$ Middle ramp setting significantly different from high ramp setting at $p \leq 0.017$

${ }^{\mathrm{d}}$ Low ramp setting significantly different from high ramp setting at $p \leq 0.017$ 


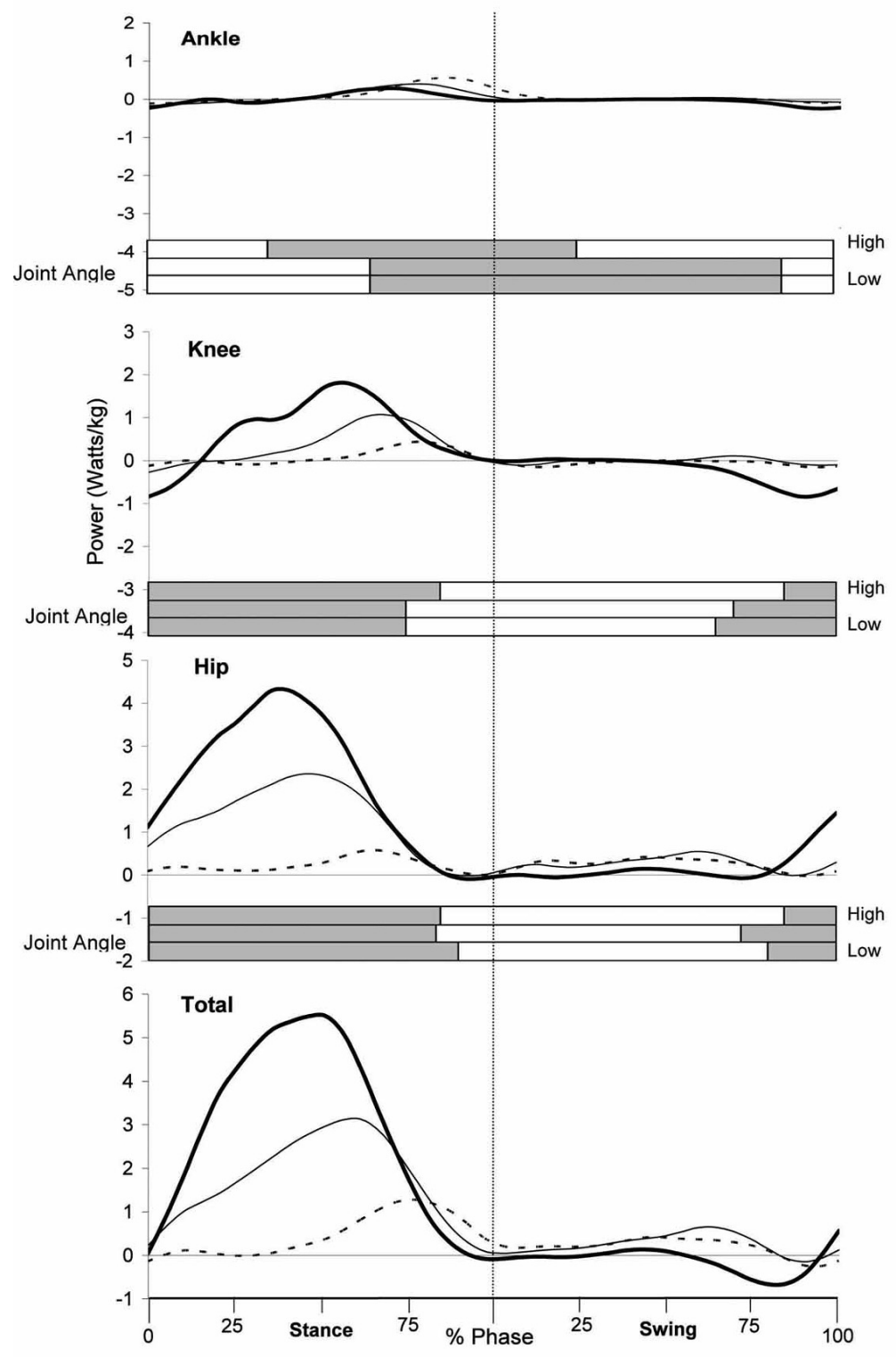

Fig. (8). Representative sample of the net joint powers for one stride cycle. Low (bold black), middle (black), and high (dashed) ramp conditions are shown. Vertical dashed line indicates change from stance to closed swing phase. Shaded bars indicate when segment is extending (or plantarflexing) at the joint, and white bars indicate when segment is flexing (or dorsiflexing) at the joint. Shade transitions indicate position of minimum and maximum joint angles.

for relative hip joint angles resemble walking at the low ramp setting [11, 14-16] and the peak hip flexion occurred in later swing phase similar to walking. The patterns of both the ankle and knee motion on the elliptical trainer are distinct from gait. The ankle relative joint angles were smaller in magnitude than those angles observed during gait. Even at the high ramp setting, relative joint angles were less than those angles required for stair ascent. At the low ramp setting, the magnitude of plantarflexion on the elliptical was similar to walking $[11,14,17]$; however, the timing was different. Maximum ankle plantarflexion occurred prior to the reaching the anterior pedal position during the closedchain swing phase. Unless the heel is lifted, the motion at the ankle is primarily controlled by the elliptical path it follows. The elliptical trainer does not provide a ROM for the ankle similar to running or even walking and this difference may be a result of the closed-chain nature of the elliptical stride.
Consistent with previous reports, the impact forces measured during the elliptical stride were similar to walking and much less than running. Results of this study do support the claims that joint loading is minimized during exercise on the elliptical trainer and pedal reaction forces are less than the reaction forces seen in walking. The joint reaction forces were slightly higher but in the range of those reported by Lu, Chien and Chen [4]. Vertical joint reaction forces were smaller in magnitude than walking [17] and running [18-20] for all three joints investigated. The vertical joint reaction forces were highest in the low ramp positions except at the ankle where the forces were greater in the high ramp position but this difference across the ramps was not significant. Vertical joint reaction forces at the hip, knee, and ankle in the present study were much less than those measured during walking, running, and stair climbing [20-24]. The vertical joint reaction forces at the knee during the elliptical stride 
were comparable to cycling $[25,26]$. The results of the present study confirm the claims made by elliptical trainer manufacturers that joint loading is minimized. Reducing compressive loads can minimize stress on the joint structures. The low impact of exercise on the elliptical trainer has great implications for musculoskeletal rehabilitation and exercise prescription for individuals with degenerative joint diseases.

For all three joints, the reaction forces in the anterior and posterior directions changed across the ramp conditions in opposite directions with an increase in the anterior and a decrease in the posterior joint reactions as the ramp increased. Peak anterior joint reaction forces increased by $40 \%$ across the three ramp settings, with magnitudes ranging between those reported for walking and running. Anterior joint reaction forces were higher than posterior joint reaction forces and ranged from $114 \mathrm{~N}$ to $437 \mathrm{~N}$ (.2 to .5 times $\mathrm{BW}$ ) and $8 \mathrm{~N}$ to $144 \mathrm{~N}$ ( $\sim 0$ to .2 times $\mathrm{BW}$ ), respectively. These values are comparable to $\mathrm{Lu}$, Chien and Chen who reported $0.158 \mathrm{BW}$ force directed posteriorly at the pedal during elliptical exercise [4]. At the knee, anteroposterior forces have implications for joint stability. Larger anteriorly directed joint reaction forces result from posteriorly directed forces applied to the pedal during stance phase. It was observed during the data collection sessions that the subjects anteriorly tilted their trunk with increasing ramp angle. This forward shift of the COM of the body may increase the magnitude of the anterior joint reaction forces acting at the lower extremity joints. Anteriorly directed joint reaction forces are opposed by the anterior cruciate ligament (ACL) at the knee joint and reach maximum values as the knee arrives at full extension. The mean peak magnitudes of the anterior forces observed in the current investigation were slightly greater than walking [23] and cycling [25] but less than running [20] and ascending stairs [27]. Posterior forces at the knee joint stress the posterior cruciate ligament (PCL) and are increased during activities that involve deep knee flexion like stair ascent/descent. The posterior joint reaction force was larger when the ramp setting was low as subjects pushed the pedal forward during late closed-chain swing to complete the stride cycle. The posterior forces measured were minimal compared to activities such as level walking, inclined walking, and stair climbing and were most similar to cycling, where there are relatively little posteriorly directed joint reaction forces [23, 25, 26]. Anteroposterior forces have not been extensively studied at the hip because the ball and socket joint structure provides inherent stability. These results have implications for knee rehabilitation protocols, as the high ramp setting stresses the ACL more than the low ramp setting and the low ramp setting stresses the PCL more.

The net joint moments calculated through inverse dynamics were within the ranges reported for other exercises like running, walking, and cycling. Yet, in many ways, the elliptical stride resembled cycling and stair ascent more than it resembles walking or running. Ankle plantarflexor moments increased with an increase in the ramp and ranged from 0.37 $\mathrm{Nm} / \mathrm{kg}$ to $0.41 \mathrm{Nm} / \mathrm{kg}$, resembling cycling [28-30], not gait [31]. Dorsiflexion net moments were minimal $(0.07 \mathrm{Nm} / \mathrm{kg}$ to $0.12 \mathrm{Nm} / \mathrm{kg}$ ) and decreased with an increase in ramp. This finding was expected as a result of the closed-chain path that the foot maintains throughout the stride cycle. At the knee joint, a knee flexor moment dominated in the low ramp condition and this differed from the results of Lu, Chien, and Chen [4]. The knee flexor moment decreased as the ramp increased and overall, the peak knee flexor moments resembled running at the low ramp setting, stair ascent at the middle ramp setting, and walking and cycling at the high ramp setting [28, 32-34]. The peak knee extensor moments measured during exercise on the elliptical trainer increased with the increase in ramp and resembled cycling $[26,28,30]$. Lu, Chein and Chen [4] reported knee flexor moments less than walking and knee extensor moments almost three times greater on the elliptical as compared to walking. At the high ramp setting, extensor moments calculated in the current investigation did not reach magnitudes similar to walking. A walking cadence variation and a different elliptical model/pedal system may explain the discrepant results observed. At the hip joint, the extensor moment was largest in the lower ramp condition and decreased as the ramp increased. The hip flexor moment increased with the increased ramp position to assist with the drive of the pedal vertically. While the extensor moments at the low setting resembled running, the peak hip flexor moments at the low ramp were similar to stair ascent, ranging from $5 \mathrm{Nm}$ to $86 \mathrm{Nm}$ (.1 to $1.4 \mathrm{Nm} / \mathrm{kg}$ ) [35]. At the high ramp settings, both the peak knee flexor and extensor moments resemble cycling while peak hip flexor moments resembled walking. Magnitudes were within the range of moments measured during various exercises. In contrast to running where knee moments are almost twice as large as hip moments, the hip joint moments were nearly as high as the net moments observed for the knee joint during the elliptical stride at the low ramp setting [19].

Joint power analysis reflects the net effect of a joint moment on the mechanical energy of the whole body [31]. It does not, however, explain the role of a muscle group in changes in the energy level of individual body segments. While the effect of energy transfer that occurs locally could be greater in magnitude or even occurring in the opposite direction, the estimation of net joint powers, when combined with knowledge of joint moments and position still provides useful information regarding the effect of the ramp settings on lower extremity mechanics during the elliptical stride. Power analyses revealed that significantly more energy was generated at the low ramp setting. Distinct from gait [15], the contribution of the ankle joint to the total power of the body was minimal during the elliptical stride. These results are not surprising, given that the plantarflexors are not utilized for propulsion during the closed-chain motion of the elliptical stride. Peak power absorption at the ankle was consistent with values reported for walking, race walking, and fast walking $[15,36,37]$. The peak power generation at the knee most closely resembled walking at the middle and high ramp setting [15, 38] and stair ascent at the low ramp setting [15]. At the low ramp setting, the knee generates power during stance, as the knee is extending. The observed joint kinetics were similar to those expected while pulling the leg backwards during exercise on a cross-country ski simulator. The knee absorbs energy during late closed-chain swing phase as the knee begins to extend and the pedal moves from the 
highest position to the lowest position. Some subjects displayed energy absorption near the posterior pedal position at the high ramp setting. The representative subject chosen shows this pattern (Fig. 8). Taken together, a flexor moment occurring during knee extension, absorbing energy, suggests eccentric muscle action of the knee extensors during the late closed-chain swing phase. The hip plays an important role in power generation during the elliptical stride across all three ramp settings. At the low ramp setting, the power generation at the hip was much greater than for walking, jogging, stair climbing and cycling [14-15, 36, 39]. At the high ramp setting, the hip generated power that was comparable in magnitude to walking [14-15]. Many subjects displayed a pattern illustrated in Fig. (8) with two positive hip joint power peaks at the high ramp setting. The first peak suggests concentric hip extensor muscle action during stance phase and the second suggests concentric hip flexor action during the closedchain swing phase. In subjects that did not display energy generation at the hip during the closed-chain swing phase, even at the high ramp setting, it can be hypothesized that these subjects used different strategies to bring the swing leg through such as shifting body weight more to the stance leg or flexion of the leg at the knee joint. The energy distribution produced during the elliptical stride across the lower extremity joints is distinct from walking and running. While peak power is generated at the ankle joint during the propulsive phase of gait, the majority of the power comes from the hip during exercise on the elliptical trainer. As the ramp setting increased, the subjects were able to utilize gravitational forces during elliptical striding, requiring less energy generation at the lower extremity joints.

\section{CONCLUSION}

This report of the observed moments and powers produced by the internal and external forces during the elliptical stride provides useful information for exercise prescription and musculoskeletal rehabilitation. If the elliptical trainer is used for cross-training in endurance events like running, athletic trainers and coaches should consider the minimal work done by the plantarflexors during exercise on the elliptical trainer. Additionally, plantarflexor strength is often reduced in older adults and exercise prescription should address this limitation of the elliptical trainer when working in special populations like older adults. While acknowledging the various limitations of inverse dynamics modeling, the experimental design, and transferability of results to different models of elliptical trainers (particularly when the arm levers are included), information presented in this study about how the lower extremities are utilized during the elliptical stride is useful to exercise enthusiasts, fitness professionals, and rehabilitation specialists. It will be important to recognize the differences in exercising on an elliptical at different ramp settings given the changes in the joint biomechanics seen at each ramp where there are similarities to walking at the low ramp settings but more similarities to cycling in the higher ramp settings.

\section{ACKNOWLEDGEMENTS}

The authors would like to thank Precor, Inc. for the use of the instrumented pedals for this project.

\section{CONFLICT OF INTEREST}

One of the authors has done contract work for Precor, Inc. This project was not funded by Precor.

\section{FUNDING}

There was no funding received for this work. The instrumented pedals used in the study were provided by Precor, Inc.

\section{REFERENCES}

[1] Hoeberigs JH. Factors related to the incidence of running injuries. A review. Sports Med 1992; 13: 408-22.

[2] Dalleck LC, Kravitz L, Robergs RA. Maximal exercise testing using the elliptical cross-trainer and treadmill. J Exerc PhysiolOnline 2004; 7: 94-101.

[3] Mercer JA, Dufek JS, Bates BT. Analysis of peak oxygen consumption and heart rate during elliptical and treadmill exercise. J Sport Rehabil 2001; 10: 48-56.

[4] Lu TW, Chien HL, Chen HL. Joint Loading in the lower extremities during elliptical exercise. Med Sci Sports Exerc 2007; 39: 1651-8.

[5] Lu TW, Chien HL. Dynamic analysis of the lower extremities during elliptical exercise. J Biomech 2006; 39: S193.

[6] Porcari JP, Zedaker JM, Naser L, Miller M. Evaluation of an elliptical exerciser in comparison to treadmill walking and running, stationary cycling, and stepping. Med Sci Sports Exerc 1998; 30: S168.

[7] Chien HL, Tsai, TY, Lu TW. The effects of pedal rates on pedal reaction forces during elliptical exercise. Biomed Eng Appl Basis Comm 2007; 4: 207-14.

[8] Dempster WT. Space requirements of the seated operator: Geometrical, kinematic, and mechanical aspects of the body with special reference to the limbs. WADC Technical Report 1955; 55-159.

[9] Robertson DGE, Caldwell GE, Hamill J, Kamen G, Whittlesey SN. Research Methods in Biomechanics. Champaign (IL): Human Kinetics 2004; 309p.

[10] Burnfield JM, Shu Y, Buster T, Taylor A. Similarity of joint kinematics and muscle demands between elliptical training and walking: Implications for practice. Phys Ther 2010; 90: 289-305.

[11] Murray MP, Drought AB, Kory RC. Walking patterns of normal men. J Bone Joint Surg Am 1964; 46: 335-60.

[12] Novacheck TF. The biomechanics of running. Gait Posture 1998; 7: 77-95.

[13] Houtz SJ, Fischer FJ. An analysis of muscle action and joint excursion during exercise on a stationary bicycle. J Bone Joint Surg Am 1959; 41: 123-31.

[14] Nordin M, Frankel VH. Basic Biomechanics of the Musculoskeletal System 3rd ed. Baltimore (MD): Lippincott Williams \& Wilkins 2001; p. 467

[15] Reiner R, Rabuffetti M, Frigo C. Stair ascent and descent at different inclinations. Gait Posture 2002; 15: 32-44.

[16] Rowe PJ, Myles CM, Walker C, Nutton R. Knee joint kinematics in gait and other functional activities measured using flexible electrogoniometry: how much knee motion is sufficient for normal daily life? Gait Posture 2000; 12: 143-55.

[17] Stauffer RN, Chao EYS, Brewster RL. Force and motion analysis of the normal, diseased, and prosthetic ankle joints. Clin Orthop Relat Res 1977; 127: 189-96.

[18] Burdett RG. Forces predicted at the ankle during running. Med Sci Sports Exerc 1982; 14: 308-16.

[19] Harrison RN, Lees A, McCullagh PJ, Rowe WB. A bioengineering analysis of human muscle and joint forces in the lower limbs during running. J Sports Sci 1986; 4: 201-18.

[20] Scott SH, Winter DA. Internal forces of chronic running injury sites. Med Sci Sports Exerc 1990; 22: 357-69.

[21] Anderson FC, Pandy MG. Static and dynamic optimization solutions for gait are practically equivalent. J Biomech 2001; 34: 153-61.

[22] Morrison JB. Function of the knee joint in various activities. J Biomed Eng 1969; 4: 573-80.

[23] Taylor WR, Heller MO, Bergmann G, Duda GN. Tibio-femoral loading during human gait and stair climbing. J Orthop Res 2004; 22: $625-32$. 
[24] van den Bogert AJ, Read L, Nigg BM. An analysis of hip joint loading during walking, running, and skiing. Med Sci Sports Exerc 1999; 31: 131-42.

[25] Ericson MO, Nisell R. Tibiofemoral joint forces during ergometer cycling. Am J Sports Med 1986; 14: 285-90.

[26] Reiser RF, Broker JP, Peterson ML. Knee loads in the standard and recumbent cycling positions. Biomed Sci Instrum 2004; 40: 36-42.

[27] Taylor SJG, Walker PS, Perry JS, Cannon SR, Woledge R. The forces in the distal femur and the knee during walking and other activities measured by telemetry. J Arthroplasty 1998; 13: 428-37.

[28] Caldwell GE, Hagberg JM, McCole SD, Li L. Lower extremity joint moments during uphill cycling. J Appl Biomech 1999; 15: 166-81.

[29] Gregor SM, Perell KL, Rushatakankovit S, Miyamoto E, Muffoletto R, Gregor R J. Lower extremity general muscle moment patterns in healthy individuals during recumbent cycling. Clin Biomech 2002; 17: 123-9.

[30] Perell KL, Gregor RJ, Kim G, Rushatakankovit S, Scremin E, Seymour L. Comparison of cycling kinetics during recumbent bicycling in subjects with and without diabetes. J Rehabil Res Dev 2002; 39: 13-20.

[31] Siegel KL, Kepple TM, Stanhope SJ. Joint moment control of mechanical energy flow during normal gait. Gait Posture 2004; 19: 69-75.
[32] Besier TF, Lloyd DG, Cochrane JD, Ackland TR. External loading of the knee joint during running and cutting maneuvers. Med Sci Sports Exerc 2001; 33: 1168-75.

[33] Costigan PA, Deluzio KJ, Wyss UP. Knee and hip kinetics during normal stair climbing. Gait Posture 2002; 16: 31-7.

[34] Ericson MO, Bratt A, Nisell R, Nemeth G, Eckholm J. Load moments about the hip and knee joints during ergometer cycling. Scand J Rehabil Med 1986; 18: 165-72.

[35] Nadeau S, McFadyen BJ, Malouin F. Frontal and sagittal plane analyses of the stair climbing task in healthy adults aged over 40 years: what are the challenges compared to level walking? Clin Biomech 2003; 18: 950-9.

[36] White SC, Winter DA. Mechanical power analysis of the lower limb musculature in race walking. Int J Sport Biomech 1985; 1: $15-24$.

[37] Winter DA, Eng P. Energy generation and absorption at the ankle and knee during fast, natural, and slow cadences. Clin Orthop Relat Res 1983; 175: 147-54.

[38] Umberger BR, Martin PE. Mechanical power and efficiency of level walking with different stride rates. J Exp Biol 2007; 210: 3255-65.

[39] Ericson MO, Batte AL, Nisell R, Arborelius UP, Ekholm J. Power output and work at different muscle groups during ergometer cycling. Eur J Appl Physiol 1986; 55: 229-35.

This is an open access article licensed under the terms of the Creative Commons Attribution Non-Commercial License (http://creativecommons.org/licenses/ by-nc/3.0/) which permits unrestricted, non-commercial use, distribution and reproduction in any medium, provided the work is properly cited. 